\title{
Neural Substrates of Depression and Resilience
}

\author{
Ming-Hu Han ${ }^{1,2}$ (D) Eric J. Nestler ${ }^{1,2,3}$
}

Published online: 10 April 2017

(C) The American Society for Experimental NeuroTherapeutics, Inc. 2017

\begin{abstract}
There is an urgent need for more effective medications to treat major depressive disorder, as fewer than half of depressed patients achieve full remission and many are not responsive with currently available antidepressant medications or psychotherapy. It is known that prolonged stressful events are an important risk factor for major depressive disorder. However, there are prominent individual variations in response to stress: a relatively small proportion of people (10-20\%) experiencing prolonged stress develop stressrelated psychiatric disorders, including depression (susceptibility to stress), whereas most stress-exposed individuals maintain normal psychological functioning (resilience to stress). There have been growing efforts to investigate the neural basis of susceptibility versus resilience to depression. An accumulating body of evidence is revealing the genetic, epigenetic, and neurophysiological mechanisms that underlie stress susceptibility, as well as the active mechanisms that underlie the resilience phenotype. In this review, we discuss,
\end{abstract}

Electronic supplementary material The online version of this article (doi:10.1007/s13311-017-0527-x) contains supplementary material, which is available to authorized users.

Ming-Hu Han

ming-hu.han@mssm.edu

$\triangle$ Eric J. Nestler

eric.nestler@mssm.edu

1 Department of Pharmacological Sciences and Institute for Systems Biomedicine, Icahn School of Medicine at Mount Sinai, New York, NY 10029, USA

2 Fishberg Department of Neuroscience and Friedman Brain Institute, Icahn School of Medicine at Mount Sinai, New York, NY 10029, USA

3 Department of Psychiatry, Icahn School of Medicine at Mount Sinai, New York, NY 10029, USA mainly based on our own work, key pathological mechanisms of susceptibility that are identified as potential therapeutic targets for depression treatment. We also review novel mechanisms that promote natural resilience as an alternative strategy to achieve treatment efficacy. These studies are opening new avenues to develop conceptually novel therapeutic strategies for depression treatment.

Keywords Major depressive disorder · Depression susceptibility $\cdot$ Resilience $\cdot$ Prefrontal cortex $\cdot$ Nucleus accumbens $\cdot$ Ventral tegmental area

\section{Introduction}

Major depressive disorder (MDD) afflicts $2 \%$ to $5 \%$ of the population worldwide, ranking first in terms of disability worldwide; and these numbers are continuously increasing [1-6]. Despite the growing population of depressed patients, fewer than half achieve full remission and many are not responsive with currently available treatments, including antidepressant medications and psychotherapies [2, 3, 5-9]. One possible reason for this situation is our incomplete understanding of the heterogeneity of the depression syndrome and the wide individual variation seen in response to prolonged or severe stress, an important risk factor for depression. The vast amount of work in this field to date has focused on investigating the pathogenic mechanisms that underlie the development of depression. In contrast, much less attention has been paid to why some individuals are psychophysiologically normal (i.e., resilient to depression), while others are not (i.e., susceptibility to depression) when they experience prolonged stress [2, 10-15]. There are growing efforts to investigate such differences because understanding the mechanisms that underlie individual variations in 
stress responses may have the unique potential to assist in the development of new therapeutic strategies for MDD.

Our group and others have used the chronic social defeat stress (CSDS) model of depression, which allows investigators to segregate susceptible and resilient subpopulations of mice [14, 16-24], to explore the molecular and neural mechanisms that underlie the individual variations in response to repeated social stress. Another advantage of the CSDS model is that susceptible mice respond to chronic, not acute, administration of standard antidepressants, as seen in humans. In this review article, we focus mainly on our own work to review several examples of mechanisms of susceptibility or of resilience, both of which provide novel drug targets for future antidepressant drug discovery.

\section{Pathological Mechanisms of Depression Susceptibility}

While serendipity has played a leading role in drug discovery efforts for depression and other psychiatric disorders [2, 3], virtually all rationale approaches until recently have been based on reversing pathogenic mechanisms. With this approach, investigators seek to identify the pathophysiological mechanisms that underlie the persistent maladaptations in an animal's brain that are induced by chronic stress or occur in genetic models of depression.

\section{Rapid Regulation of Depression-Related Behaviors}

As noted above, all approved antidepressant medications require several weeks or months of administration to achieve their therapeutic effects. For this reason, research in the field has been based on the notion that the reversal of persistent maladaptations in depressed individuals can only be achieved by chronic treatments. This approach has led to numerous hypotheses for gradual antidepressant responsivity, most notably the neurotrophic hypothesis of depression [25].

However, more recent work has provided clear evidence that mood status can be regulated rapidly in humans. For instance, Bejjani et al. [26] observed that transient acute depression was induced by high-frequency stimulation of the left subthalamic region of a patient with Parkinson's disease. The effects induced by the stimulation were seen within a time scale of several minutes (Fig. 1), suggesting unknown, rapidly acting mechanisms that control mood. Shortly thereafter it was discovered that single doses of intravenous ketamine induce rapid antidepressant effects in severely depressed individuals who failed to respond to traditional therapies [27]. This finding has since been replicated in double-blinded studies by several groups [28], confirming the rapid, bidirectional control of depression.

Consistent with these clinical studies, there is increasing evidence that optogenetically activating or inhibiting selected neural circuits and specific neuronal populations in the brain can rapidly and bidirectionally regulate depression-related behaviors in several rodent models of depression [18, 29-31]. This work has focused on several reward- and memory-related brain regions, including the medial prefrontal cortex (mPFC), ventral hippocampus (vHIP), nucleus accumbens (NAc), and ventral tegmental area (VTA).

Several studies have reported that the functioning of the mPFC is decreased in both depressed human patients and in animal models of depression. For example, Covington et al. [29] showed that molecular correlates of neuronal activity (i.e., immediate early gene expression) in $\mathrm{mPFC}$ were decreased in the postmortem tissues of clinically depressed humans, as well as in mice susceptible to CSDS. Optogenetic stimulation of $\mathrm{mPFC}$ neurons during the 5-min social interaction test in depressed susceptible mice rapidly reversed social avoidant behavior and normalized sucrose preference deficits. Similarly, Bagot et al. [32] showed that
Fig. 1 Deep brain stimulation rapidly regulates mood status. The recording time is indicated on the photograph. a Patient's usual expression while receiving levodopa. b Change in facial expression $17 \mathrm{~s}$ after stimulation began. $\mathbf{c}$ Patient crying and expressing despair $4 \mathrm{~min}$ and $16 \mathrm{~s}$ after the start of stimulation. $\mathbf{d}$ Patient laughing $1 \mathrm{~min}$ and $20 \mathrm{~s}$ after the stimulator was turned off. Adapted from Bejjani et al. [26]
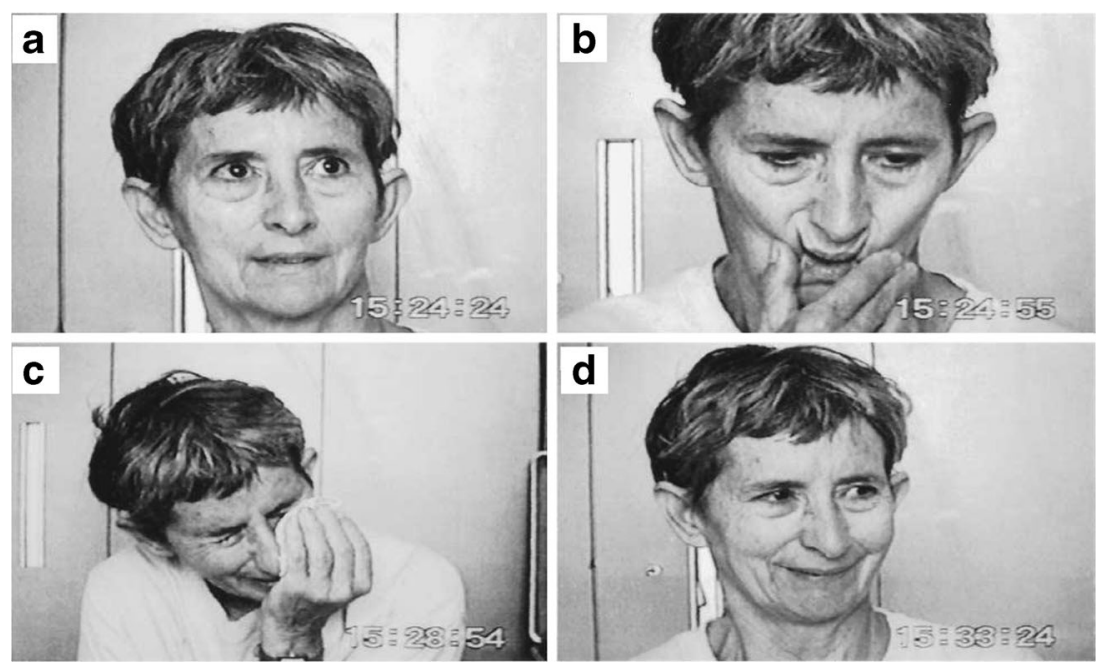
acute optogenetic stimulation of vHIP glutamatergic nerve terminals that innervate the NAc in the context of submaximal social stress induced social avoidance behavior during the 5min interaction test, whereas suppression of this specific circuit induced a rapid antidepressant-like response after CSDS.

It was hypothesized many years ago that the mesolimbic dopamine system, composed of the VTA and NAc, is a potential target for rapid antidepressant action [33]. This hypothesis has been consistently supported by more recent optogenetic studies [18-20,30]. Chaudhury et al. [18, 19] found that VTA dopamine neurons projecting to the NAc displayed hyperactivity in susceptible mice after CSDS, and that optogenetically mimicking this pathological hyperactivity of VTA-NAc dopamine neurons during the 5-min social interaction test promoted susceptible phenotypes, including social avoidant behavior and decreased sucrose preference (Fig. 2). In contrast, VTA dopamine neurons projecting to the $\mathrm{mPFC}$ exhibit hypoactivity in susceptible mice, and optogenetic inhibition of this VTA-mPFC dopamine circuit also rapidly induces social avoidance, but not decreased sucrose preference [18]. Conversely, suppression of VTA-NAc dopamine neurons, or activation of VTA-mPFC dopamine neurons, exerted proresilience effects. These findings suggest that the VTA-NAc and VTA-mPFC dopamine circuits are functionally different in rapidly inducing depression-related behaviors. Different results were obtained with a different stress model [30], suggesting further that dopaminergic systems function differently based on the type or severity of the stress involved. Interestingly, Zhang et al. [34] observed that the firing activity of locus coeruleus (LC)-norepinephrine
(NE) neurons projecting to the VTA was increased selectively in the resilient mice after CSDS. Optogenetically stimulating these LC-VTA NE neurons has no effect on the social interaction behavior during the 5-min interaction test, but stimulating these neurons $20 \mathrm{~min}$ per day for 10 days completely reverses susceptible phenotypes, including social avoidance and decreased sucrose preference. These studies suggest that the acute activation of LC-VTA NE neurons, in contrast to VTA dopamine neurons, has no rapid effect on depressionrelated behaviors.

These studies support the idea that specific integrative synaptic circuits within the brain are capable of mediating rapid effects on depression-related behaviors, either inducing those behaviors or reversing them depending on the circuit and experimental conditions involved. However, more investigations are needed to explore details about the role of individual circuits in acute regulation of mood and in validating these effects in humans. It would also be important to know whether or not these same circuits mediate the rapid antidepressant actions of ketamine.

\section{Brain-Derived Neurotrophic Factor}

Brain-derived neurotrophic factor (BDNF) is implicated in playing an important role in the pathophysiology of depression and in mediating antidepressant actions [7, 25, 35-38]. However, its pathological changes are distinct in different brain regions [7, 39]. In particular, higher levels of BDNF in the mesolimbic dopamine reward circuit are associated with a depression phenotype, which is opposite to the antidepressant-
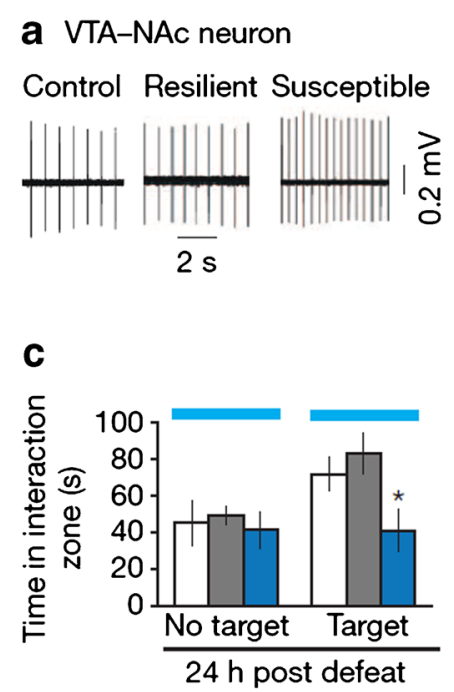

\section{b}

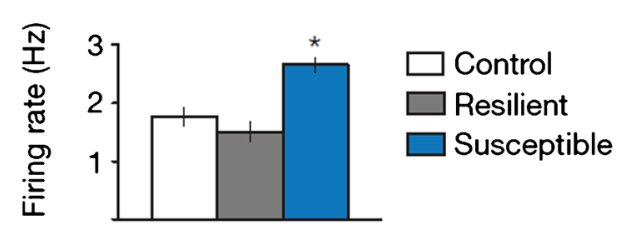

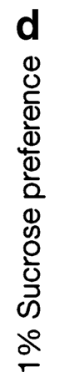

Fig. 2 Mesolimbic dopamine neurons exhibit hyperactivity in susceptible mice after chronic social defeat stress (CSDS), and optogenetic stimulation of these neurons during the 5-min social interaction test rapidly regulates depression-related behaviors post submaximal defeat. a Sample traces recorded from ventral tegmental area (VTA)-nucleus accumbens (NAc) dopamine neurons. b Firing rates of VTA-NAc dopamine neurons in control, resilient, and

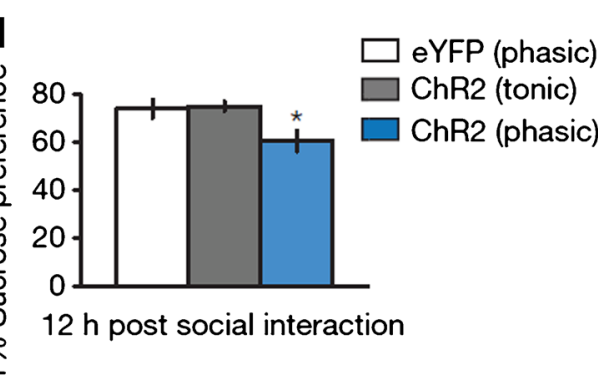

susceptible mice after CSDS. c Optical phasic but not tonic activation of VTA-NAc dopamine neurons during the 5-min interaction test induces social avoidant behavior post submaximal defeat. $\mathbf{d}$ Optical phasic but not tonic activation of VTA dopamine neurons during the 5-min interaction test decreases sucrose preference post submaximal defeat. Adapted from Chaudhury et al. [18] 
like role for BDNF in the hippocampus $[3,4,14,40]$. We focus here on the prodepression-like effect of BDNF in the VTA-NAc pathway; the reader is referred elsewhere for a review of BDNF actions in hippocampus [41].

In the CSDS model of depression, Berton and Nestler [3] reported that CSDS increased the levels of BDNF in the NAc, and showed that the VTA is the crucial source of BDNF for this increase. A later study demonstrated increased BDNF levels in NAc of susceptible but not resilient mice [14]. This BNDF change is coincident with the increase in the firing activity of VTA dopamine neurons seen in susceptible mice only (see more information below). Cao et al. [17] showed that the baseline firing rate and phasic firing properties of VTA dopamine neurons in anesthetized mice were increased selectively in susceptible mice. Interestingly, optogenetic phasic but not tonic stimulation of VTA-NAc dopamine neurons during the social interaction test induced social avoidance behavior and a sucrose preference deficit in mice exposed to submaximal social defeat stress [18]. Consistent with these behavioral data, optogenetic phasic activation of VTA-NAc dopamine neurons after such submaximal social stress induced a dramatic increase in BDNF levels in NAc, whereas tonic stimulation of these neurons was without effect [19].

Importantly, we showed recently that intra-NAc infusion of a BDNF-tyrosine receptor kinase B (TrkB) antagonist, but not dopamine receptor antagonists, prevented CSDS-induced behavioral abnormalities [31]. Local knockout of BDNF from the VTA had a similar effect. Moreover, repeated optogenetic phasic stimulation of the VTA-NAc circuit exacerbated the social defeat-induced behavioral symptoms, effects that were normalized by intra-NAc infusion of a TrkB antagonist. These findings support the view that BDNF-TrkB signaling, rather than dopamine signaling, in the VTA-NAc circuit mediates CSDS-induced depressive-like behaviors. As BDNF exerts opposite effects in different neural circuits, targeting BDNFTrkB signaling may not be the most advantageous approach for drug discovery efforts. Rather, current work is focused on identifying signaling proteins downstream of BDNF-TrkB, which might be targeted for novel therapeutics.

\section{Corticotrophin Releasing Factor}

Corticotrophin releasing factor (CRF) has long been of interest in depression research as an upstream regulator of the hypothalamic-pituitary-adrenal axis. More recent research has implicated a role for CRF in the VTA-NAc reward circuit. It is known that mesolimbic dopamine neurons respond to rewarding, as well as aversive, stimuli, and can mediate divergent behavioral outputs [14, 17, 30, 42-45]. This suggests a possible context-specific role of this mesolimbic circuit in encoding behaviors. Walsh et al. [19] replicated that optogenetic phasic stimulation of VTA-NAc dopamine neurons, in the context of submaximal social defeat stress, induced social avoidant behavior, as stated above. However, the same optogenetic stimulation of these neurons in stressnaïve mice had no effect on social interaction behavior or on NAc BDNF levels, suggesting that phasic stimulation alone is not sufficient to induce BDNF release and its behavioral sequelae.

In further work, Walsh et al. [19] demonstrated that the intra-NAc infusion of a CRF receptor antagonist blocked the phasic stimulation-induced social avoidance in mice that were subjected to submaximal defeat. In contrast, in stress-naïve mice, the combination of intra-NAc infusion of CRF plus phasic stimulation of VTA-NAc dopamine neurons increased NAc BDNF levels without affecting social behavior. These findings support the notion that CRF acting in the NAc gates the ability of phasic firing, in the context of stress, to increase BDNF signaling in the NAc and that such increased BDNF signaling promotes depression-like behavioral abnormalities but only in conjunction with other stress-induced maladaptations.

\section{Chromatin Mechanisms of Stress Susceptibility}

We have proposed that the persisting nature of behavioral abnormalities induced by CSDS and other forms of chronic stress in animal models, and by analogy in depressed patients, is mediated, in large part, through lasting changes in gene expression [46]. A corollary of this hypothesis is that such lasting changes in gene expression are mediated by stable chromatin modifications that control gene transcription. While this work remains at relatively early stages of development, growing empirical evidence supports the importance of chromatin abnormalities as key pathophysiological mechanisms of depression.

Histone deacetylases (HDACs) remove acetyl groups from several Lys residues in histone proteins, which generally promote gene expression. Open-ended, genome-wide explorations of gene expression in several brain regions have shown - surprisingly - that fewer genes are altered by CSDS in susceptible versus resilient mice [14, 24], suggesting that susceptibility may represent the failure of plasticity due to mechanisms that suppress transcriptional regulation. In support of this possibility, the direct infusion of HDAC inhibitors into any of several brain regions, including the $\mathrm{mPFC}$, vHIP, NAc, or amygdala, exert antidepressant-like effects [47-49]. In some brain regions, HDAC inhibition was more effective than standard antidepressant therapy. Systemic administration of such inhibitors are only weakly effective [37,50], but this is likely due to the poor penetration of available inhibitors into the brain. The robust efficacy of HDAC inhibitors in animal models supports the value in developing more selective inhibitors that penetrate the brain.

Another mechanism that contributes to the loss of transcriptional plasticity in depression is stress induction of 
BAZ1A, a chromatin remodeling factor that suppresses gene expression [51]. BAZ1A is induced in the NAc of depressed humans and chronically stressed mice, an effect seen in both males and females. Overexpression of BAZ1A in NAc of mouse models promotes stress susceptibility, whereas its knockdown in this brain region promotes resilience and also reverses depression-related behavioral abnormalities induced by CSDS. While drugs that inhibit BAZ1A directly are likely to be too broadly acting to be safe in treating depression, targeting the specific genes that are suppressed by BAZ1A in the depressed state would be a viable path for antidepressant discovery efforts.

Chromatin mechanisms are highly complex and it would be a mistake to view all suppressive mechanisms as prodepressant. An example of this complexity is G9a, a histone methyltransferase that methylates certain Lys residues on the histone $\mathrm{H} 3$ subunit, a modification that promotes suppression of gene expression. Levels of G9a and its methylated histone mark are reduced in NAc of depressed humans and chronically stressed mice [52]. Local knockdown of G9a in this brain region promotes susceptibility in stressed mice, whereas overexpression of G9a causes the opposite effect. Interestingly, one of the major pathways affected by G9a and its histone-methylating effects is BDNF-TrkB signaling: loss of G9a in NAc in the depressed state leads to increased expression levels of proteins downstream of TrkB, which ultimately lead to increased activation of the transcription factor, cAMP response element binding protein [52]. cAMP response element binding protein, like BDNF, exerts different effects on depression-related abnormalities, promoting depression in NAc but opposing depression in hippocampus [7].

\section{A New Therapeutic Strategy: Targeting Mechanisms of Resilience}

According to the American Psychological Association (2014), resilience is defined as "the process of adapting well in the face of adversity, trauma, tragedy, threats or even significant sources of stress". Increasing evidence supports the view that promoting mechanisms of natural resilience represents a bone fide alternative path for generating novel antidepressant treatments.

\section{Active Resilience}

It has become clear that resilience is an active stress-coping mechanism, rather than a simple lack of pathological alterations in response to prolonged adverse stimuli. Many psychosocial coping skills, including facing fears, optimism, cognitive reappraisal, and purpose of life, have long been used successfully in daily life [53]. Recent studies have begun to reveal the neurobiological basis for resilience mechanisms
$[54,55]$. Active coping behaviors, such as escape and aggression, are associated with more transient activation of the hypothalamus-pituitary-adrenal axis [56]. Consistent with this, overexpression of CRF in mutant mice increases active coping behavior, a phenomenon that is mediated partly by enhanced noradrenergic activity [57]. In contrast, positive emotions and mutual cooperation are linked to the function of the mesolimbic reward neural circuit [58].

Studies with the CSDS model are consistent with this view of active resilience. As noted above, genome-wide measures of gene expression changes show greater plasticity in most brain regions of resilient mice as compared with susceptible mice; this is illustrated for the VTA and NAc in Fig. 3. Additionally, the firing rate and bursting events of VTA dopamine neurons - as assessed in both in vitro brain slice work and in vivo electrophysiological studies - is increased in susceptible mice, but not in the resilient subgroup as discussed earlier $[14,17]$. Subsequent work demonstrated that the ability of resilient mice to escape this pathological maladaptation is based on their ability to mount additional mechanisms of plasticity that normalize VTA function. These observations establish the VTA as a useful model system in which to understand molecular and cellular mechanisms of active resilience.

\section{KCNQ Potassium Channels as Novel Drug Targets}

VTA dopamine neurons respond differentially to distinct stressors [30, 59-63]. Studies from several groups showed that CSDS induces a hyperactivity of VTA dopamine neurons in susceptible mice [14, 17, 20, 60-62, 64]. Moreover, VTA dopamine neurons are heterogeneous with respect to their projection pathways. As stated earlier, in susceptible mice, VTA-NAc dopamine neurons display hyperactivity, whereas VTA-mPFC dopamine neurons exhibit decreased firing. In contrast to susceptible mice, the resilient group has a control-level firing activity in both of these projection circuits [18-20].

Mechanistically, in susceptible mice, the VTA-NAc dopamine neurons display an increased excitatory $I_{\mathrm{h}}$ current (hyperpolarization-activated cation channel current), which drives the pathological hyperactivity of these neurons [17, 20]. Unexpectedly, in the resilient group, the VTA-NAc dopamine neurons have an even larger excitatory $I_{\mathrm{h}}$ current, which is observed in parallel with increased inhibitory $\mathrm{K}^{+}$ channel currents. This finding suggests that a homeostatic $\mathrm{E} / \mathrm{I}$ current balance stabilizes the firing activity and maintains decreased excitability of VTA-NAc dopamine cells in the resilient phenotype [20, 55, 65] (Fig. 4). This neurophysiological work demonstrates that resilience uses more ion channelmediated currents to cope actively with stress, which is consistent with previous molecular studies showing more genes regulated in the resilient group [14, 24, 54]. In particular, there are 4 different subtypes of $\mathrm{K}^{+}$channels, including $\mathrm{KCNQ}$ 
a
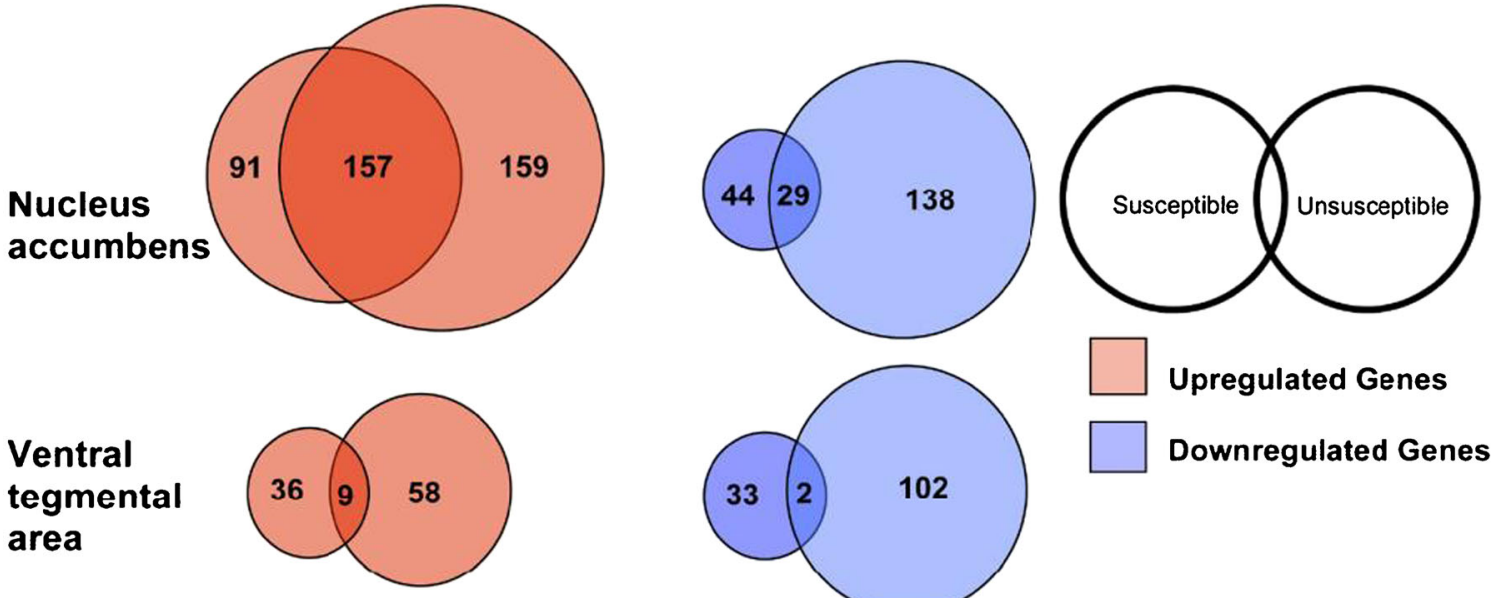

Downregulated Genes

b

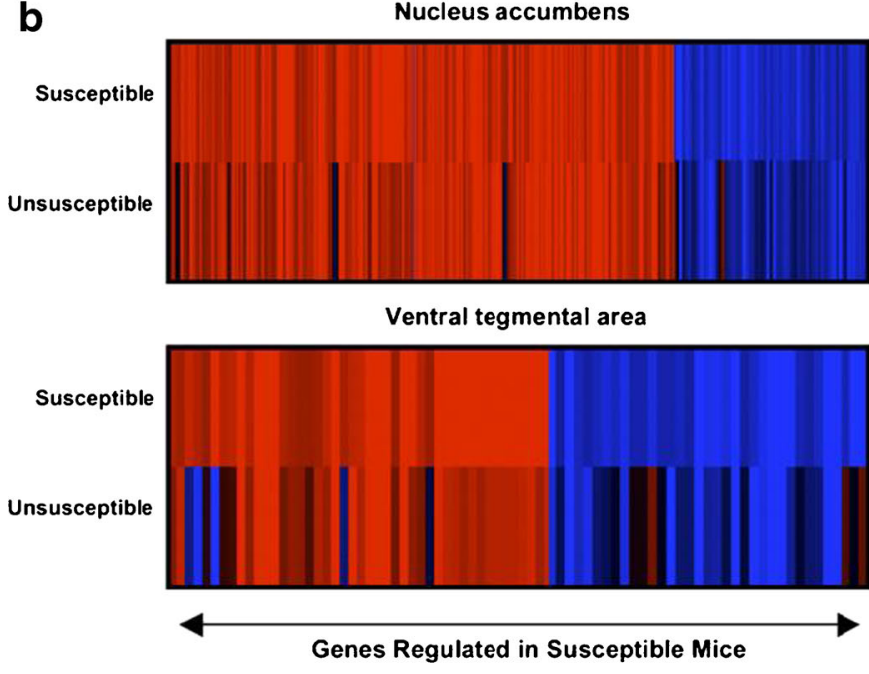

Fig. 3 Resilience is an active process of adapting to adverse stimuli, rather than a passive lack of maladaptations. a Microarray data show that $>300$ and 80 genes are regulated in nucleus accumbens (NAc) and ventral tetmental area (VTA), respectively, of susceptible mice, as compared with stress-naïve handled controls. By contrast, many more genes (482 in the NAc and 171 in the VTA) are regulated in the

channels, selectively upregulated in the VTA of resilient mice [14].

This insight into the molecular and cellular basis of natural resilience as an active stress-coping process offers a new therapeutic direction. Friedman et al. [20] demonstrated that KCNQ channel function was significantly upregulated in the VTA-NAc dopamine neurons of resilient mice, with no effect seen in susceptible mice. KCNQ overexpression in these neurons of the susceptible subgroup normalized their pathologic neuronal hyperactivity and reversed the animals' depressionlike behavioral abnormalities. Furthermore, KCNQ "openers" (potentiators), applied through either local infusion into the VTA or systemic administration, completely reversed the depressive-like symptoms displayed by susceptible animals. Ezogabine (retigabine) is a nonselective KCNQ potentiator

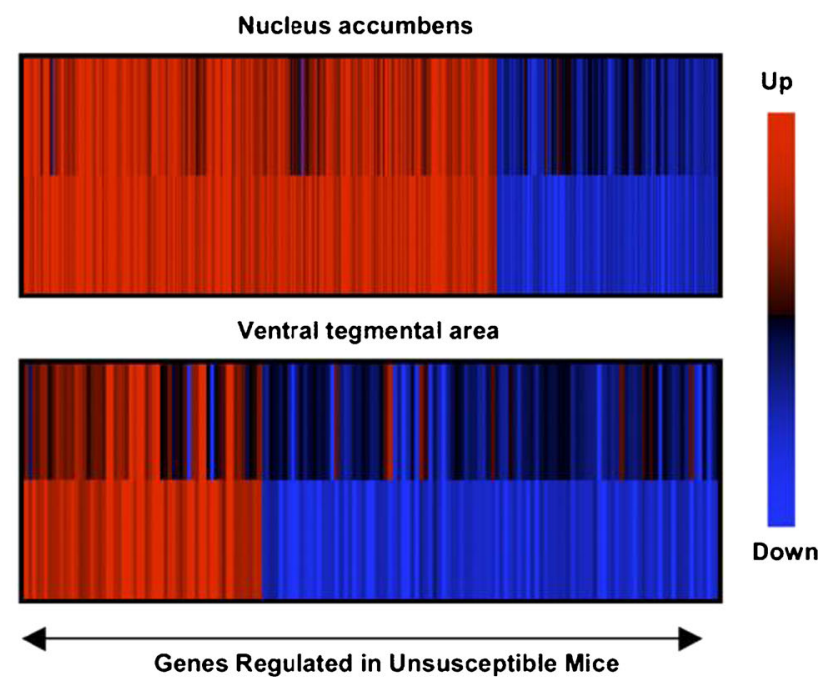

resilient (unsusceptible) subpopulation. b Heat maps illustrate the regulation of genes in the NAc and VTA of susceptible and resilient mice, with red-to-blue gradient depicting an upregulation to downregulation ( $\geq 2$-fold increase $\rightarrow \leq 2$-fold decrease). In each panel, top and bottom rows show significantly regulated genes in susceptible and resilient mice, respectively. Adapted from Krishnan et al., [14]

and is approved by the approved for the treatment of epilepsy. Systemically administrated ezogabine normalized both VTA neuronal hyperactivity and depression-like behaviors seen in susceptible mice [21]. These findings provided the rationale for a small scale clinical trial of ezogabine in treatmentresistant depressed patients who exhibit prominent anhedonia symptoms and reduced functional magnetic resonance imaging signals in the NAc. Ezogabine reversed both of these abnormalities [66], thus setting the stage for a larger clinical trial now underway. Together, this work identifies KCNQ as a target for conceptually novel antidepressants that function through the potentiation of active resilience mechanisms. Moving forward it would be important to generate more selective KCNQ potentiators that target channel subtypes enriched in VTA. 
a

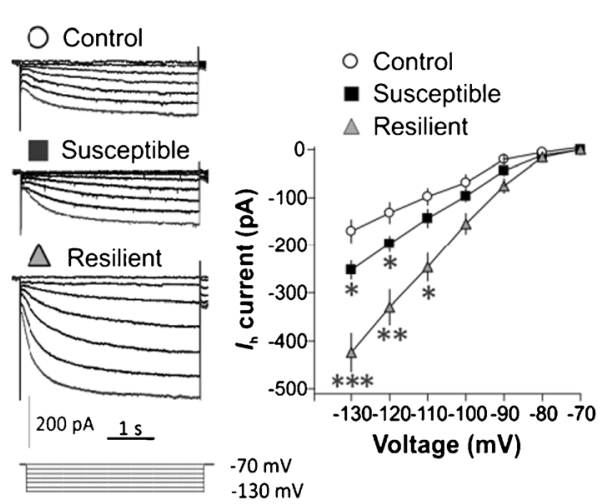

b

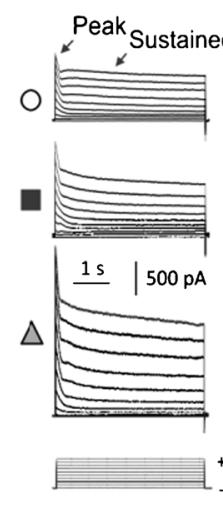

$\mathrm{K}^{+}$

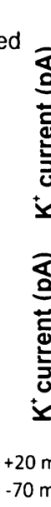

Fig. 4 Active ion-channel mechanisms that underlie resilience. a $I_{\mathrm{h}}$ sample traces and statistical data for control (open circles), susceptible (filled black squares), and resilient (filled gray triangles) mice. Post hoc analysis at $-130 \mathrm{mV}$ shows a significant increase in $I_{\mathrm{h}}$ in susceptible mice and an even significantly larger $I_{\mathrm{h}}$ increase in the resilient subgroup compared with the control. b Sample traces and statistical data of isolated $\mathrm{K}^{+}$channel-mediated currents recorded from VTA dopamine neurons show that resilient mice have significantly increased peak and

\section{NE Mechanisms of Resilience}

Emerging evidence implicates the LC-NE system in stress resilience [67-69]. It is well known that the LC-NE nucleus responds to stress by "priming" neurons throughout the brain $[68,70-76]$. For instance, in response to acute stress, the LCNE system shifts the brain into a rapid defense-mechanism state [77]. An increasing number of studies has also demonstrated the heterogeneous principles of organization and operation of LC-NE neurons, as seen in the VTA dopaminergic system. Previous evidence showed that LC neurons innervate the VTA [78-80].

Consistent with these early studies, Zhang et al. [34] found recently that, in the CSDS paradigm, resilient mice displayed an increased firing activity of LC neurons that project to the VTA, and that repeated optogenetic activation of LC-VTA NE neurons (20 min per day for 10 days) in susceptible mice reversed depression-like behaviors and pathological hyperactivity of VTA-NAc dopamine neurons as stated above. Utilizing a circuit-mapping molecular profiling technique, this study screened the molecular targets of LC noradrenergic inputs onto VTA-NAc dopamine neurons and found a higher expression of $\alpha 1 \mathrm{~b}$ and $\beta 3$ adrenoceptors in VTA-NAc dopamine neurons. Further investigation revealed that repeated intra-VTA infusion of $\alpha 1$ and $\beta 3$ agonists for 10 days normalized social avoidance behavior and pathological hyperactivity of VTA-NAc dopamine neurons, which is consistent with the 10-day optical stimulation-induced effects [34]. Consistent with these findings, Isingrini et al. [81] reported that elevated NE release was observed in the VTA of resilient animals. These highly consistent findings implicate the potential role of NE in mediating resilience mechanisms in the VTA.

C Excitability

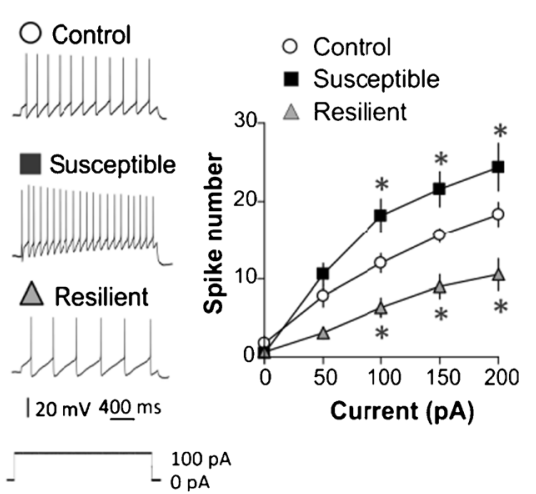

sustained phases of $\mathrm{K}^{+}$currents. Post hoc analysis shows a slight increase in peak $\mathrm{K}^{+}$currents in susceptible mice and a larger increase in the resilient subgroup compared with the controls. c Susceptible mice display increased dopamine neuron excitability, whereas resilient mice display a reduction, after incremental steps in current injections (50, 100,150 , and $200 \mathrm{pA}$ ), compared with controls. Post hoc analysis of susceptible and resilient compared with control mice for 100,150, and $200 \mathrm{pA}$ currents at $p<0.05$. Adapted from Friedman et al. [20]

Current research is focusing on further characterizing the NE receptor(s) that mediate these proresilience actions, with the notion that agonists at those receptors might represent another approach for antidepressant therapy.

\section{Transcriptional Mechanisms of Resilience}

There have also been efforts to identify the transcription factors that drive the considerable degree of transcriptional plasticity that occurs throughout the brain in resilient animals. One of the best characterized is $\Delta \mathrm{FosB}$, a truncated product of the FosB immediate early gene. $\triangle \mathrm{FosB}$ is unique among immediate early gene products in that it is a stable protein, which accumulates in a region-specific manner in brain in response to many types of chronic perturbations and thereby mediates lasting transcriptional effects [82]. $\Delta$ FosB is most studied in drug abuse models, where its induction in NAc in response to chronic drug administration promotes drug reward and a drugaddicted state. More recent research has demonstrated the importance of $\triangle \mathrm{FosB}$ in stress models as well [83]. $\triangle \mathrm{FosB}$ is induced selectively in D1-type medium spiny neurons of the NAc in resilient mice after CSDS, where its overexpression has been shown to promote resilience and prevention of its induction promoted susceptibility $[84,85]$. Such induction of $\triangle \mathrm{FosB}$ in the NAc of resilient animals is mediated by another transcription factor, serum response factor, which also promoted resilience in this brain region [86].

Another proresilient transcription factor is $\beta$-catenin, which is downstream of WNT signaling and implicated in numerous cellular functions. WNT signaling pathways are among the most highly regulated in the NAc of resilient animals after CSDS based on genome-wide studies of gene 
expression [87]. This action appears to be selective for D2type medium spiny neurons [22]. Consistent with these findings, overexpression of $\beta$-catenin in D2 neurons of NAc promoted resilience, whereas its knockdown in this region promoted susceptibility [22].

A major challenge of this research is how to translate these results into the clinic. Transcription factors are not common drug targets for psychiatric syndromes, although they are increasingly targeted in other fields of medicine (e.g., cancer). It is also argued that drugs that target transcription factors have the additional impediment of having to penetrate the cell and nucleus; however, most drugs that penetrate the blood-brain barrier also penetrate cell and nuclear membranes. A more compelling obstacle is that most transcription factors are broadly expressed, such that drugs that target them are likely to cause many off-target effects. This is particularly the case for $\beta$-catenin, which is expressed ubiquitously and whose activation systemically would be expected to cause cancer. For this reason, many transcription factors should not be viewed as drug targets themselves, but as ways of identifying the key genes through which they produce their behavioral effects, with the products of those genes representing bona fide drug targets. For example, we and others have mapped transcription factor targets in the brain by use of chromatin immunoprecipitation followed by deep sequencing (ChIPseq) (e.g., [22]). The results of this study revealed numerous gene targets whose protein products could conceivably serve as the basis for future drug discovery efforts.

Nevertheless, $\Delta$ FosB may be an exception to the notion that transcription factors are not easily druggable. It is not expressed broadly in the brain or periphery such that drugs that directly target $\Delta$ FosB might be viable therapeutically. Nevertheless, a final challenge with this approach is the diversity of $\Delta$ FosB actions in different neuronal cell types. While $\Delta$ FosB exerts proresilient and antidepressant-like effects when activated in D1 NAc neurons, it does the opposite when activated in D2 NAc neurons [85]. It also does the opposite when induced in $\mathrm{mPFC}$ pyramidal neurons [88]. Therefore, the only way to assess the therapeutic potential of $\Delta \mathrm{FosB}$ is to develop tool compounds that can be given systemically and either inhibit or activate $\Delta$ FosB. The lack of availability of such tool compounds to $\Delta \mathrm{FosB}$ and a range of other novel targets is one of the greatest hurdles in central nervous system drug discovery.

\section{Conclusion}

The enormous impact of depression on humanity, and limitations in currently available therapies, make it imperative to develop more effective treatments for this syndrome. Most work in the field over several decades has focused on ways to reverse the deleterious effects of chronic stress in animal models. This remains, of course, a viable approach, even though we have, to date, failed to introduce antidepressants with novel mechanisms of action. One explanation for this failure is that many of the adaptations that occur in genetically normal animals in response to chronic stress do not mediate the deleterious effects of the stress, but rather represent adaptive mechanisms that serve to help the individual cope positively with the stress. A crucial innovation in recent years is the ability to distinguish between susceptible versus resilient animals subjected to chronic stress. Antidepressant drug discovery efforts will thus be helped moving forward by efforts to reverse mechanisms demonstrated specifically to promote susceptibility. Additionally, another innovation is to understand mechanisms of natural resilience with the novel idea that promoting such mechanisms in more susceptible individuals represents a novel path for antidepressant drug discovery. Indeed, in recent years, many new genetic, epigenetic and neurophysiological mechanisms of stress susceptibility and resilience have been revealed and validated in animal models, with several now being tested in the clinic. We remain hopeful that these new avenues offer conceptually original therapeutic strategies for depression treatment.

Acknowledgements This work was supported by grants from the National Institute of Mental Health (M.H.H., R21MH112081; E.J.N., R01MH051399, P50MH096890), the National Institute of Alcohol Abuse and Alcoholism (M.H.H., R01AA022445), the Brain and Behavior Research Foundation (M.H.H., NARSAD), and the Hope for Depression Research Foundation (E.J.N.).

Required Author Forms Disclosure forms provided by the authors are available with the online version of this article.

\section{References}

1. Kessler RC, McGonagle KA, Zhao S, et al. Lifetime and 12-month prevalence of DSM-III-R psychiatric disorders in the United States. Results from the National Comorbidity Survey. Arch Gen Psychiatry 1994;51:8-19.

2. Nestler EJ, Barrot M, DiLeone RJ, Eisch AJ, Gold SJ, Monteggia LM. Neurobiology of depression. Neuron 2002;34:13-25.

3. Berton O, Nestler EJ. New approaches to antidepressant drug discovery: beyond monoamines. Nat Rev Neurosci 2006;7:137-151.

4. Krishnan V, Nestler EJ. The molecular neurobiology of depression. Nature 2008;455:894-902.

5. Ferrari AJ, Charlson FJ, Norman RE, et al. The epidemiological modelling of major depressive disorder: application for the Global Burden of Disease Study 2010. PLOS ONE 2013;8:e69637.

6. Ferrari AJ, Charlson FJ, Norman RE, et al. Burden of depressive disorders by country, sex, age, and year: findings from the global burden of disease study 2010. PLOS Med 2013;10:e1001547.

7. Nestler EJ, Carlezon WA, Jr. The mesolimbic dopamine reward circuit in depression. Biol Psychiatry 2006;59:1151-1159.

8. Nestler EJ, Gould E, Manji H. Preclinical models: status of basic research in depression. Biol Psychiatry 2002;52:503-528.

9. Toseeb U, Brage S, Corder K, et al. Exercise and depressive symptoms in adolescents: a longitudinal cohort study. JAMA Pediatr 2014;168:1093-1100. 
10. Southwick SM, Vythilingam M, Charney DS. The psychobiology of depression and resilience to stress: implications for prevention and treatment. Annu Rev Clin Psychol 2005;1:255-291.

11. Charney DS, Manji HK. Life stress, genes, and depression: multiple pathways lead to increased risk and new opportunities for intervention. Sci STKE 2004;2004:re5.

12. Yehuda R, Flory JD, Southwick S, Charney DS. Developing an agenda for translational studies of resilience and vulnerability following trauma exposure. Ann N Y Acad Sci 2006;1071:379-396.

13. Yehuda R. Risk and resilience in posttraumatic stress disorder. J Clin Psychiatry 2004;65:29-36.

14. Krishnan V, Han MH, Graham DL, et al. Molecular adaptations underlying susceptibility and resistance to social defeat in brain reward regions. Cell 2007;131:391-404.

15. Atkinson PA, Martin CR, Rankin J. Resilience revisited. J Psychiatric Ment Health Nurs 2009;16:137-145.

16. Krishnan V, Han MH, Mazei-Robison M, et al. AKT signaling within the ventral tegmental area regulates cellular and behavioral responses to stressful stimuli. Biol Psychiatry 2008;64:691-700.

17. Cao JL, Covington HE, 3rd, Friedman AK, et al. Mesolimbic dopamine neurons in the brain reward circuit mediate susceptibility to social defeat and antidepressant action. J Neurosci 2010;30:1645316458 .

18. Chaudhury D, Walsh JJ, Friedman AK, et al. Rapid regulation of depression-related behaviours by control of midbrain dopamine neurons. Nature 2013;493:532-536.

19. Walsh JJ, Friedman AK, Sun H, et al. Stress and CRF gate neural activation of BDNF in the mesolimbic reward pathway. Nat Neurosci 2014;17:27-29.

20. Friedman AK, Walsh JJ, Juarez B, et al. Enhancing depression mechanisms in midbrain dopamine neurons achieves homeostatic resilience. Science 2014;344:313-319.

21. Friedman AK, Juarez B, Ku SM, et al. KCNQ channel openers reverse depressive symptoms via an active resilience mechanism. Nat Commun 2016;7:11671.

22. Dias C, Feng J, Sun H, et al. beta-catenin mediates stress resilience through Dicer1/microRNA regulation. Nature 2014;516:51-55.

23. Golden SA, Covington HE, 3rd, Berton O, Russo SJ. A standardized protocol for repeated social defeat stress in mice. Nat Protoc 2011;6:1183-1191.

24. Bagot RC, Cates HM, Purushothaman I, et al. Circuit-wide transcriptional profiling reveals brain region-specific gene networks regulating depression susceptibility. Neuron 2016;90:969-983.

25. Duman RS, Heninger GR, Nestler EJ. A molecular and cellular theory of depression. Arch Gen Psychiatry 1997;54:597-606.

26. Bejjani BP, Damier P, Arnulf I, et al. Transient acute depression induced by high-frequency deep-brain stimulation. N Engl J Med 1999;340:1476-1480.

27. Berman RM, Cappiello A, Anand A, et al. Antidepressant effects of ketamine in depressed patients. Biol Psychiatry 2000;47:351-354.

28. Niciu MJ, Henter ID, Luckenbaugh DA, Zarate CA, Jr., Charney DS. Glutamate receptor antagonists as fast-acting therapeutic alternatives for the treatment of depression: ketamine and other compounds. Annu Rev Pharmacol Toxicol 2014;54:119-139.

29. Covington HE, 3rd, Lobo MK, Maze I, et al. Antidepressant effect of optogenetic stimulation of the medial prefrontal cortex. J Neurosci 2010;30:16082-16090.

30. Tye KM, Mirzabekov JJ, Warden MR, et al. Dopamine neurons modulate neural encoding and expression of depressionrelated behaviour. Nature 2013;493:537-541.

31. Wook Koo J, Labonte B, Engmann O, et al. Essential role of mesolimbic brain-derived neurotrophic factor in chronic social stress-induced depressive behaviors. Biol Psychiatry 2016;80: 469-478.
32. Bagot RC, Parise EM, Pena CJ, et al. Ventral hippocampal afferents to the nucleus accumbens regulate susceptibility to depression. Nat Commun 2015;6:7062

33. Willner P. The mesolimbic dopamine system as a target for rapid antidepressant action. Int Clin Psychopharmacol 1997;12(Suppl. 3):S7-S14.

34. Zhang H, Chaudhury D, Juarez B, et al. Role of locus coeruleusventral tegmental area circuit in mediating the resilience to social stress. Neuropsychopharmacology 2015;2015:S237-S238.

35. Krishnan V, Nestler EJ. Linking molecules to mood: new insight into the biology of depression. Am J Psychiatry 2010;167:13051320.

36. Monteggia LM, Barrot M, Powell CM, et al. Essential role of brainderived neurotrophic factor in adult hippocampal function. Proc Natl Acad Sci U S A 2004;101:10827-10832.

37. Tsankova NM, Berton O, Renthal W, Kumar A, Neve RL, Nestler EJ. Sustained hippocampal chromatin regulation in a mouse model of depression and antidepressant action. Nat Neurosci 2006;9:519525.

38. Monteggia LM, Luikart B, Barrot M, et al. Brain-derived neurotrophic factor conditional knockouts show gender differences in depression-related behaviors. Biol Psychiatry 2007;61:187-197.

39. Schmidt HD, Duman RS. The role of neurotrophic factors in adult hippocampal neurogenesis, antidepressant treatments and animal models of depressive-like behavior. Behav Pharmacol 2007;18: 391-418.

40. Eisch AJ, Bolanos CA, de Wit J, et al. Brain-derived neurotrophic factor in the ventral midbrain-nucleus accumbens pathway: a role in depression. Biol Psychiatry 2003;54:994-1005.

41. Duman RS, Monteggia LM. A neurotrophic model for stressrelated mood disorders. Biol Psychiatry 2006;59:1116-1127.

42. Grace AA, Floresco SB, Goto Y, Lodge DJ. Regulation of firing of dopaminergic neurons and control of goal-directed behaviors. Trends Neurosci 2007;30:220-227.

43. Lammel S, Ion DI, Roeper J, Malenka RC. Projection-specific modulation of dopamine neuron synapses by aversive and rewarding stimuli. Neuron 2011;70:855-862.

44. Koo JW, Mazei-Robison MS, Chaudhury D, et al. BDNF is a negative modulator of morphine action. Science 2012; 338:124-128.

45. Wanat MJ, Bonci A, Phillips PE. CRF acts in the midbrain to attenuate accumbens dopamine release to rewards but not their predictors. Nat Neurosci 2013;16:383-385.

46. Vialou V, Feng J, Robison AJ, Nestler EJ. Epigenetic mechanisms of depression and antidepressant action. Annu Rev Pharmacol Toxicol 2013;53:59-87.

47. Covington HE, 3rd, Maze I, LaPlant QC, et al. Antidepressant actions of histone deacetylase inhibitors. J Neurosci 2009;29: 11451-11460.

48. Covington HE, 3rd, Vialou VF, LaPlant Q, Ohnishi YN, Nestler EJ. Hippocampal-dependent antidepressant-like activity of histone deacetylase inhibition. Neurosci Lett 2011;493:122-126.

49. Covington HE, 3rd, Maze I, Vialou V, Nestler EJ. Antidepressant action of HDAC inhibition in the prefrontal cortex. Neuroscience 2015;298:329-335.

50. Schroeder FA, Lin CL, Crusio WE, Akbarian S. Antidepressantlike effects of the histone deacetylase inhibitor, sodium butyrate, in the mouse. Biol Psychiatry 2007;62:55-64.

51. Sun H, Damez-Werno DM, Scobie KN, et al. ACF chromatinremodeling complex mediates stress-induced depressive-like behavior. Nat Med 2015;21:1146-1153.

52. Covington HE, 3rd, Maze I, Sun H, et al. A role for repressive histone methylation in cocaine-induced vulnerability to stress. Neuron 2011;71:656-670.

53. Southwick SM, Charney DS. Resilience: The Science of Mastering Life's Greatest Challenges: Cambridge University Press. 2012. 
54. Feder A, Nestler EJ, Charney DS. Psychobiology and molecular genetics of resilience. Nat Rev Neurosci 2009;10:446-457.

55. Russo SJ, Murrough JW, Han MH, Charney DS, Nestler EJ. Neurobiology of resilience. Nat Neurosci 2012;15:1475-1484.

56. Korte SM, Koolhaas JM, Wingfield JC, McEwen BS. The Darwinian concept of stress: benefits of allostasis and costs of allostatic load and the trade-offs in health and disease. Neurosci Biobehav Rev 2005;29:3-38.

57. Lu A, Steiner MA, Whittle N, et al. Conditional mouse mutants highlight mechanisms of corticotropin-releasing hormone effects on stress-coping behavior. Mol Psychiatry 2008;13:1028-1042.

58. Vythilingam M, Nelson EE, Scaramozza M, et al. Reward circuitry in resilience to severe trauma: an fMRI investigation of resilient special forces soldiers. Psychiatry Res 2009;172:75-77.

59. Moore H, Rose HJ, Grace AA. Chronic cold stress reduces the spontaneous activity of ventral tegmental dopamine neurons. Neuropsychopharmacology 2001;24:410-419.

60. Anstrom KK, Miczek KA, Budygin EA. Increased phasic dopamine signaling in the mesolimbic pathway during social defeat in rats. Neuroscience 2009;161:3-12.

61. Razzoli M, Andreoli M, Michielin F, Quarta D, Sokal DM. Increased phasic activity of VTA dopamine neurons in mice 3 weeks after repeated social defeat. Behav Brain Res 2011;218: 253-257.

62. Valenti O, Gill KM, Grace AA. Different stressors produce excitation or inhibition of mesolimbic dopamine neuron activity: response alteration by stress preexposure. Eur J Neurosci 2012;35:13121321.

63. Chang $\mathrm{CH}$, Grace AA. Amygdala-ventral pallidum pathway decreases dopamine activity after chronic mild stress in rats. Biol Psychiatry 2014;76:223-230.

64. Greenberg GD, Steinman MQ, Doig IE, Hao R, Trainor BC. Effects of social defeat on dopamine neurons in the ventral tegmental area in male and female California mice. Eur J Neurosci 2015;42:30813094.

65. Walsh JJ, Han MH. The heterogeneity of ventral tegmental area neurons: projection functions in a mood-related context. Neuroscience 2014;282C:101-108.

66. van Dam NT, Kautz M, Friedman AK, et al. Potassium channel modulator ezogabine decreases symptomatology and increases reward response in depression. Annual Meeting of Society of Biological Psychiatry; 2016 SOBP Abstracts In press; Atlanta, Georgia.

67. Krystal JH, Neumeister A. Noradrenergic and serotonergic mechanisms in the neurobiology of posttraumatic stress disorder and resilience. Brain Res 2009;1293:13-23.

68. Charney DS. Psychobiological mechanisms of resilience and vulnerability: implications for successful adaptation to extreme stress. Am J Psychiatry 2004;161:195-216.

69. Valentino RJ, Van Bockstaele E. Endogenous opioids: the downside of opposing stress. Neurobiol Stress 2015;1:23-32.

70. Valentino RJ, Foote SL, Page ME. The locus coeruleus as a site for integrating corticotropin-releasing factor and noradrenergic mediation of stress responses. Ann N Y Acad Sci 1993;697:173-188.
71. Koob GF. Corticotropin-releasing factor, norepinephrine, and stress. Biol Psychiatry 1999;46:1167-1180.

72. Nestler EJ, Alreja M, Aghajanian GK. Molecular control of locus coeruleus neurotransmission. Biol Psychiatry 1999;46:1131-1139.

73. Reyes BA, Bangasser DA, Valentino RJ, Van Bockstaele EJ. Using high resolution imaging to determine trafficking of corticotropinreleasing factor receptors in noradrenergic neurons of the rat locus coeruleus. Life Sci 2014;112:2-9.

74. Bingham B, McFadden K, Zhang X, Bhatnagar S, Beck S, Valentino R. Early adolescence as a critical window during which social stress distinctly alters behavior and brain norepinephrine activity. Neuropsychopharmacology 2011;36:896-909.

75. McCall JG, Al-Hasani R, Siuda ER, et al. CRH Engagement of the locus coeruleus noradrenergic system mediates stress induced anxiety. Neuron 2015;87:605-620.

76. Nestler EJ, Aghajanian GK. Molecular and cellular basis of addiction. Science 1997;278:58-63.

77. Hermans EJ, van Marle HJ, Ossewaarde L, et al. Stress-related noradrenergic activity prompts large-scale neural network reconfiguration. Science 2011;334:1151-1153.

78. Paladini CA, Williams JT. Noradrenergic inhibition of midbrain dopamine neurons. J Neurosci 2004;24:4568-4575.

79. Arencibia-Albite F, Paladini C, Williams JT, Jimenez-Rivera CA. Noradrenergic modulation of the hyperpolarization-activated cation current (Ih) in dopamine neurons of the ventral tegmental area. Neuroscience 2007;149:303-314.

80. Sara SJ. The locus coeruleus and noradrenergic modulation of cognition. Nat Rev Neurosci 2009;10:211-223.

81. Isingrini E, Perret L, Rainer Q, et al. Resilience to chronic stress is mediated by noradrenergic regulation of dopamine neurons. Nat Neurosci 2016;19:560-563.

82. Robison AJ, Nestler EJ. Transcriptional and epigenetic mechanisms of addiction. Nat Rev Neurosci 2011;12:623-637.

83. Nestler EJ. FosB: a transcriptional regulator of stress and antidepressant responses. Eur J Pharmacol 2015;753:66-72.

84. Vialou V, Robison AJ, Laplant QC, et al. DeltaFosB in brain reward circuits mediates resilience to stress and antidepressant responses. Nat Neurosci 2010;13:745-752.

85. Lobo MK, Zaman S, Damez-Werno DM, et al. DeltaFosB induction in striatal medium spiny neuron subtypes in response to chronic pharmacological, emotional, and optogenetic stimuli. J Neurosci 2013;33:18381-18395.

86 Vialou V, Maze I, Renthal W, et al. Serum response factor promotes resilience to chronic social stress through the induction of DeltaFosB. J Neurosci 2010;30:14585-14592.

87 Wilkinson MB, Dias C, Magida J, et al. A novel role of the WNTdishevelled-GSK3beta signaling cascade in the mouse nucleus accumbens in a social defeat model of depression. J Neurosci 2011;31: 9084-9092.

88 Vialou V, Bagot RC, Cahill ME, et al. Prefrontal cortical circuit for depression- and anxiety-related behaviors mediated by cholecystokinin: role of DeltaFosB. J Neurosci 2014;34:3878-3887. 